\title{
Identification and in vitro Characterization of a Novel Phage Endolysin that Targets Gram-Negative Bacteria
}

\author{
Jaewoo Bai ${ }^{1,2,3}$, Sangmi Lee ${ }^{4}$ (D) and Sangryeol Ryu $1,2,5,6, * \mathbb{D}$ \\ 1 Department of Food and Animal Biotechnology, Seoul National University, Seoul 08826, Korea; \\ bbbjjjwww@snu.ac.kr \\ 2 Department of Agricultural Biotechnology, Seoul National University, Seoul 08826, Korea \\ 3 Division of Applied Food System, Major in Food Science \& Technology, Seoul Women's University, \\ Seoul 01797, Korea; jwbai@swu.ac.kr \\ 4 Department of Food and Nutrition, Chungbuk National University, Cheongju, Chungbuk 28644, Korea; \\ sangmilee@chungbuk.ac.kr \\ 5 Research Institute of Agriculture and Life Sciences, Seoul National University, Seoul 08826, Korea \\ 6 Center for Food and Bioconvergence, Seoul National University, Seoul 08826, Korea \\ * Correspondence: sangryu@snu.ac.kr
}

Received: 8 March 2020; Accepted: 18 March 2020; Published: 21 March 2020

check for updates

\begin{abstract}
Most double-stranded (ds) DNA phages utilize holin proteins to secrete endolysin for host peptidoglycan lysis. In contrast, several holin-independent endolysins with secretion sequences or signal-arrest-release (SAR) sequences are secreted via the Sec pathway. In this study, we characterized a novel lysis protein (M4Lys) encoded by the dsDNA phage BSPM4, whose lysis function is not dependent on either holin or the Sec pathway in vitro. In silico analysis of M4Lys revealed that it contains a putative virion protein domain and an unusual C-terminal transmembrane domain (TMD). Turbidity reduction assays and liquid chromatography-mass spectrometry using purified peptidoglycan showed that the virion protein domain of M4Lys has peptidoglycan lysis activity. In vitro overproduction of M4Lys in Escherichia coli revealed that M4Lys alone caused rapid cell lysis. Treatment of E. coli with a Sec inhibitor did not inhibit the lysis activity of M4Lys, indicating that the Sec pathway is not involved in M4Lys-mediated cell lysis. Truncation of the TMD eliminated the cell lysis phenomenon, while production of the TMD alone did not induce the cell lysis. All these findings demonstrate that M4Lys is a novel endolysin that has a unique mosaic structure distinct from other canonical endolysins and the TMD plays a critical role in M4Lys-mediated in vitro cell lysis.
\end{abstract}

Keywords: cell lysis kinetics; endolysin; flagella-targeting phage; transmembrane domain; secretion

\section{Introduction}

Bacteriophages rely on diverse cell lysis mechanisms to release their progeny from host bacteria. Bacterial lysis by phages can be accomplished in at least two different ways, i.e., peptidoglycan degradation by endolysins or the inhibition of peptidoglycan synthesis [1-3]. The majority (96\%) of phages described to date are tailed phages that contain a double stranded (ds) DNA genome. Most of these phages achieve host cell lysis by degrading peptidoglycan via the holin-endolysin system while single-stranded RNA or DNA phages inhibit peptidoglycan synthesis $[4,5]$.

Endolysins are peptidoglycan hydrolases that disintegrate integrity of the cell wall by cleaving the peptidoglycan. It is known that endolysins generally cleave glycosidic bonds, peptide bonds, or amide bonds of the peptidoglycan. Holins are small membrane proteins that dissipate membrane potential or make holes in the membrane to allow endolysins to access the peptidoglycan target $[4,6]$. In the holin-endolysin system, endolysins are produced and accumulate in the cytoplasm in the late stage of 
the phage lytic cycle. Canonically, holins form holes in the cytoplasmic membrane at a specific time, allowing the endolysin to access peptidoglycan substrates. Peptidoglycan degradation by endolysin results in osmotic imbalance and subsequent rapid cell lysis [7].

In contrast, some endolysins are exported via holin-independent mechanisms. For example, the endolysins of phages P1, 21, and phiKMV, which have N-terminal signal-arrest-release (SAR) domains, are secreted to the periplasm by the Sec pathway. The SAR domain-containing endolysins are anchored to the cytoplasmic membrane in an inactive form until holins dissipate the membrane proton motive force [8-10]. The endolysin of Oenococcus oeni phage fOg44 (Lys44) contains a typical signal sequence that is converted into the active form after cleavage of the $\mathrm{N}$-terminal signal peptide. The $\mathrm{N}$-terminal region of Lys44 functions as an export signal for the endolysin to translocate to the periplasmic region [11]. The above-mentioned endolysins access the periplasmic region through non-cleavable $\mathrm{N}$-terminal hydrophobic regions or cleavable signal sequences, which function as secretion sequences, and are translocated via Sec machinery. In addition, it has been reported that an auxiliary lysis protein, Gp1, of mycobacteriophage Ms6 is required for efficient host cell lysis by its endolysin, LysA. Gp1 acts as a chaperone-like protein, which is involved in LysA delivery to the peptidoglycan [12].

In this study, we characterized a novel endolysin, M4Lys, identified from the flagella-targeting bacteriophage BSPM4 that was isolated by our group and whose whole genome sequence has been analyzed (accession no. KY620117) [13]. In vitro expression of M4Lys alone in Escherichia coli induced rapid cell lysis. The Sec pathway was not responsible for M4Lys-mediated cell lysis in vitro while a C-terminal transmembrane domain (TMD) was indispensable for this cell lysis. Additional cell lysis kinetics studies using various truncations of M4Lys also indicated that the TMD can play an important role for M4Lys-mediated lysis. All these findings suggest that M4Lys can induce cell lysis without the help of holin when it is overproduced in vitro.

\section{Materials and Methods}

\subsection{In Silico Analysis of the BSPM4 Phage Lysis Cassette}

The genome of Salmonella enterica serovar Typhimurium phage BSPM4 was sequenced and annotated previously [13]. The complete genome sequence and annotation results of S. Typhimurium phage BSPM4 are available in GenBank under accession number KY620117 [13]. Domains of M4Lys endolysin were analyzed using BLASTp [14], InterProScan [15], the National Center for Biotechnology Information (NCBI) conserved domain database (CDD) [16], and Phobius [17].

\subsection{Bacterial Strains, Media, and Growth Conditions}

The bacterial strains and plasmids used in this study are listed in Table 1. Bacterial strains were grown in Luria-Bertani medium (Difco, Detroit, MI, USA) at $37^{\circ} \mathrm{C}$ with aeration. Escherichia coli DH5 $\alpha$ and BL21 (DE3) strains were used for cloning and protein expression, respectively. Appropriate antibiotics [ $50 \mu \mathrm{g} / \mathrm{mL}$ ampicillin (Amp) or $50 \mu \mathrm{g} / \mathrm{mL}$ kanamycin (Kan), final concentration] were used if necessary. When indicated, isopropyl- $\beta$-thiogalactopyranoside (IPTG) and sodium azide $\left(\mathrm{NaN}_{3}\right)$ were added at $0.1 \mathrm{mM}$ and $1-10 \mathrm{mM}$, respectively. 
Table 1. Bacterial strains and plasmids in this study.

\begin{tabular}{|c|c|c|}
\hline Strains & Description & Reference \\
\hline \multicolumn{3}{|l|}{ Escherichia coli } \\
\hline $\mathrm{DH} 5 \alpha$ & $\begin{array}{c}\mathrm{F}^{-} \text {\$80lacZ } \Delta \mathrm{M} 15 \Delta\left(\text { lacZYA-argF) U169 recA1 endA1 hsdR17( } \mathrm{r}_{\mathrm{k}}^{-}\right. \\
\mathrm{m}_{\mathrm{k}}^{+} \text {) phoA supE44 thi-1 gyrA96 relA1 } \lambda^{-}\end{array}$ & Invitrogen \\
\hline BL21 (DE3) & $\mathrm{F}-\mathrm{ompT} h \mathrm{hdSB}\left(\mathrm{r}_{\mathrm{B}}-\mathrm{m}_{\mathrm{B}}-\right)$ gal dcm (DE3) & [18] \\
\hline \multicolumn{3}{|c|}{ 2. } \\
\hline pET28a $(+)$ & Expression vector with a hexahistidine tag, $\mathrm{Kan}^{\mathrm{r}}$ & Novagen \\
\hline pETDuet-1 & Dual-expression vector with a hexahistidine tag, $\mathrm{Amp}^{\mathrm{r}}$ & Novagen \\
\hline pM4Lys $\Delta \mathrm{TMD}$ & pET28a-orf38 $\Delta T M D_{1-206}$ & This study \\
\hline M4Lys & pETDuet-1-orf38 & This study \\
\hline ORF_37 & pETDuet-1-orf37 & This study \\
\hline M4Lys-ORF_37 & pETDuet-1-orf37.orf 38 & This study \\
\hline M4Lys $\Delta \mathrm{TMD}$ & pETDuet-1-orf38 $\Delta T M D_{1-206}$ & This study \\
\hline M4Lys $\Delta$ TMD-ORF_37 & pETDuet-1- orf38 $T_{M M D_{1-206}} \cdot$ orf37 & This study \\
\hline M4TMD & pETDuet-1-orf38TMD & This study \\
\hline M4Lys $\Delta$ TMD-TMD & pETDuet-1- orf $38 \Delta T M D_{1-206} \cdot T M D_{158-237}$ & This study \\
\hline M4Lys $1-226$ & pET28a-orf38 $1-226$ & This study \\
\hline M4Lys $_{1-225}$ & pET28a-orf $388_{1-225}$ & This study \\
\hline M4Lys $_{1-224}$ & pET28a-orf $381-224$ & This study \\
\hline
\end{tabular}

\subsection{Cloning and Expression of the Lysis Proteins}

The putative endolysin gene (ORF_38, M4Lys) was amplified by polymerase chain reaction (PCR) with the BSPM4Lys-F-NdeI and BSPM4Lys-R-XhoI primers using phage BSPM4 DNA as template. The PCR product was digested with the respective restriction enzymes and cloned into the pETDuet-1 vector (Novagen, Madison, WI, USA) and sequenced. The recombinant plasmids were, then, used to transform E. coli BL21 (DE3) strain and induced to examine cell lysis kinetics. The putative holin gene (ORF_37, M4Holin) was PCR-amplified with the BSPM4Holin-F-NcoI and BSPM4Holin-R-HindIII primers and cloned into the pETDuet- 1 as described above. To coexpress M4Lys and M4Holin, both genes were cloned into pETDuet-1 using the primers above. To produce the proteins, $0.1 \mathrm{mM}$ of IPTG was added $3 \mathrm{~h}$ after incubation and the lysis kinetics of each strain was observed as previously described [8]. Cell lysis activity of M4Lys was further confirmed with the addition of various concentrations of IPTG (0.0-1.0 mM). Experiments for the lysis kinetics were performed in triplicate. The non-recombinant pETDuet-1 plasmid-harboring E. coli BL21 (DE3) strain was used as a control. All the primers used for PCR amplification are listed in Table 2. 
Table 2. Primers used in this study.

\begin{tabular}{ccc}
\hline Primer & ${\text { Nucleotide Sequence [5'-3'] }{ }^{+}}^{\text {Restriction Site }^{\prime}}$ \\
\hline BSPM4Lys-F-NdeI & ATG AGG AAA TAA CAT ATG GCT AAA CAG AAG & NdeI \\
BSPM4Lys-R-XhoI & GAA AAC GAG CGC CTC GAG GAC GCC CGT CTT & XhoI \\
BSPM4Lys2-R-XhoI & GGC CGC GCC GAA CTC GAG GCG TAC TCA ATC ACC & XhoI \\
BSPM4Lys5_-R-XhoI & CTT CCG GTA CTC GAG TCA TCC CAT GTA CAG & Xhol \\
BSPM4Lys54-R-XhoI & CCG ATG CTT CTC GAG CGC TCA CCA TCC CAT & XhoI \\
BSPM4Lys6-R-XhoI & CCC GGC CCG ATG CTC GAG GTA CTA GAC CCA TCC & XhoI \\
BSPM4orf37-F-NcoI & CCC GCT AAT TTT TTG TGA GGA CCA TGG GC A TGA GCG & NcoI \\
& AAA TGG AAC G & HindIII \\
BSPM4orf37-R-HindIII & GTT ATT GCG AAT CCC GCG AAG CTT CTG TTT AGC CAT & NcoI \\
pETDuet-M4TMD-NcoI-F & CGA GGA AGG TCT GCC CAT GGG GGG CAT CGT TAA G & HindIII \\
pETDuet-M4TMD-HindIII-R & AAA ACG AGC GCC GCA AGC TTG CCC GTC TTG ATC C & - \\
Duet-UP1-F & GAT GCG TCC GGC GTA GAG G & - \\
Duet-DOWN1-R & CGA TTA TGC GGC CGT GTA CAA T & - \\
Duet-UP2-F & ATT GTA CAC GGC CGC ATA ATC G & - \\
T7-promoter-F & TAATACGACTCACTATAGGG & - \\
T7-terminator-R & GCT AGT TAT TGC TCA GCG GTG & \\
\hline
\end{tabular}

$\dagger$, Restriction enzyme sites are underlined.

\subsection{Overproduction and Purification of the M4Lys $\triangle T M D$ Protein}

To purify a soluble form of M4Lys protein lacking the C-terminal TMD (designated as M4Lys $\triangle T M D$ ), the M4Lys $\triangle T M D$ coding gene was cloned into the pET28a vector using the BSPM4Lys-F-NdeI and BSPM4Lys2-R-XhoI primers. In the cloning experiments to generate recombinant plasmids, DNA fragments encoding the putative endolysin gene were inserted between the NdeI and XhoI restriction sites of the pET28a plasmid resulting in an N-terminal His-tag. The recombinant plasmid-harboring E. coli BL21 (DE3) strain was incubated for $2 \mathrm{~h}$ until an optical density at $600 \mathrm{~nm}$ $\left(\mathrm{OD}_{600}\right)$ of 0.5 was reached and was, then, supplemented with $0.5 \mathrm{mM} \mathrm{IPTG}$ to produce the M4Lys $\triangle \mathrm{TMD}$ protein. After incubation for $3 \mathrm{~h}$, cells were suspended in lysis buffer $(20 \mathrm{mM}$ Tris-Cl, $200 \mathrm{mM} \mathrm{NaCl}$, $\mathrm{pH}$ 8.0) and sonicated (Branson Ultrasonics, Danbury, CT, USA) to break bacterial cell walls. Sonicated cells were centrifuged at $15,000 \times g$ for $20 \mathrm{~min}$ to obtain the supernatant containing soluble proteins. His-tagged M4Lys $\triangle T M D$ was purified using a Ni-nitrilotriacetic acid Superflow column (Qiagen $\mathrm{GmbH}$, Hilden, Germany) according to the manufacturer's instructions. The purified protein was visualized with sodium dodecyl sulfate (SDS)-polyacrylamide gel electrophoresis (PAGE) and stored in storage buffer (20 mM Tris-Cl, $200 \mathrm{mM} \mathrm{NaCl}, 30 \%$ glycerol, $\mathrm{pH}$ 8.0) after exchanging buffers using a PD Miditrap G-25 column (GE healthcare, Bucks, UK) [19].

\subsection{Lytic Activity and Host Range Test}

Lytic activity of purified M4Lys $\triangle$ TMD was determined using purified E. coli peptidoglycan (muropeptides) as substrates. Crude muropeptides of E. coli BL21 (DE3) were extracted as described previously with slight modifications [20,21]. In brief, overnight grown E. coli cells were harvested and the cells were disrupted by sonication. The cell suspension was then centrifuged at $1400 \times g$ for $10 \mathrm{~min}$ to obtain the crude cell wall lysate. Muropeptides were pelleted by centrifugation at $27,000 \times g$ for $5 \mathrm{~min}$, resuspended in $4 \%$ SDS solution, and boiled for $10 \mathrm{~min}$. Muropeptides were washed using cold distilled water at least 3 times, suspended in Tris- $\mathrm{HCl}$ buffer $(\mathrm{pH} 8.0)$, and stored at $4{ }^{\circ} \mathrm{C}$ until use. Peptidoglycan lysis activity of M4Lys $\triangle T M D$ was examined by measuring a reduction in $\mathrm{OD}_{600}$ of the muropeptide solution [21]. To determine the host range, lawns of autoclaved cells of different strains were prepared in 24-well plates. Then, $1 \mathrm{mM}$ of purified M4Lys $\Delta \mathrm{TMD}$ was spotted on each lawn and incubated at $37^{\circ} \mathrm{C}$ for $12 \mathrm{~h}$. $\mathrm{OD}_{600}$ was measured using a SpectraMax i3x (Molecular Devices, Silicon Valley, CA, USA) microplate reader. The reduction of turbidity was calculated by comparing with that of the respective control group without enzyme treatment. The bacterial strains used for host range 
analysis are listed in Table 3. The efficiency of plating (EOP) was calculated by the comparison of titers between the selected bacteria and the propagation host strain S. Typhimurium LT2C [22].

Table 3. Determination of host range of BSPM4 bacteriophage and its endolysin.

\begin{tabular}{|c|c|c|}
\hline Bacterial Strain & $\begin{array}{l}\text { BSPM4 } \\
\text { (phage) }^{*}\end{array}$ & $\begin{array}{l}\text { M4Lys } \Delta T M D \\
\text { (endolysin) }^{\#}\end{array}$ \\
\hline \multicolumn{3}{|l|}{$\begin{array}{l}\text { Salmonella enterica } \\
\text { serovar Typhimurium }\end{array}$} \\
\hline SL1344 & $\mathrm{CC}$ & ++ \\
\hline UK1 & $\mathrm{CCC}$ & ++ \\
\hline LT2 & $\mathrm{C}$ & + \\
\hline LT2C & $\mathrm{C}$ & + \\
\hline ATCC $14028^{\dagger}$ & $\mathrm{C}$ & ++ \\
\hline ATCC19586 & $\mathrm{C}$ & + \\
\hline ATCC 43147 & $\mathrm{CCC}$ & + \\
\hline ATCC13076 & $\mathrm{C}$ & ++ \\
\hline DT104 & $\mathrm{C}$ & + \\
\hline \multicolumn{3}{|l|}{ serovar Paratyphi } \\
\hline A IB 211 & - & + \\
\hline B IB 231 & - & ++ \\
\hline serovar Dublin IB 2973 & - & ++ \\
\hline \multicolumn{3}{|l|}{ E. coli } \\
\hline BL21 & - & +++ \\
\hline \multicolumn{3}{|l|}{ E. coliO157:H7 } \\
\hline ATCC35150 & - & +++ \\
\hline ATCC 43890 & - & + \\
\hline ATCC43894 & - & +++ \\
\hline ATCC43895 & - & ++ \\
\hline O157:NM 3204-92 & - & ++ \\
\hline O157:NM H-0482 & - & + \\
\hline \multicolumn{3}{|l|}{ Gram-negative bacteria } \\
\hline Vibrio fischeri ES-114 ATCC 700601 & - & - \\
\hline Pseudomonas aeruginosa ATCC 27853 & - & +++ \\
\hline Cronobacter sakazakii ATCC29544 & - & + \\
\hline Gram-positive bacteria & & \\
\hline Staphylococcus aureus ATCC 29213 & - & - \\
\hline Staphylococcus epidermis ATCC 35983 & - & - \\
\hline Bacillus subtilis ATCC 23857 & - & - \\
\hline Bacillus cereus ATCC 14579 & - & - \\
\hline
\end{tabular}

†, ATCC, American Type Culture Collection. *, CCC, efficiency of plating (EOP) of 2 to 1.5 ; CC, EOP of 1.5 to 1 ; C, EOP of 1 to $0.5 ;-$, not susceptible to phages. \#, Degree of OD reduction; +++, 0.4 to $0.6 ;++, 0.3$ to $0.4 ;+, 0.2$ to 0.3 ; -, no reduction.

\subsection{Target Site Identification of M4Lys with HPLC}

Muropeptides for HPLC analysis was prepared as described above with slight modifications [23]. Briefly, $400 \mathrm{~mL}$ of E. coli MG1655 cells were grown exponentially $\left(\mathrm{OD}_{578}=0.6\right)$ and harvested by centrifugation at $12,000 \times \mathrm{g}$ for $15 \mathrm{~min}$ at $4{ }^{\circ} \mathrm{C}$. The cell pellet was resuspended in $3 \mathrm{~mL}$ of ice-cold deionized water (D.W.) and $3 \mathrm{~mL}$ of boiling $8 \%$ SDS was added dropwise with vigorous stirring within $10 \mathrm{~min}$. The mixture was boiled for $30 \mathrm{~min}$ and incubated overnight at room temperature. Muropeptides were collected by ultracentrifugation $(130,000 \times g, 60 \mathrm{~min}$ at room temperature). The pellet was washed 4 times with $8 \mathrm{~mL}$ of D.W. to remove residual SDS. High molecular weight glycogen and covalently bound lipoprotein were cleaved by adding $\alpha$-amylase $(100 \mu \mathrm{g} / \mathrm{mL}$ in $10 \mathrm{mM}$ Tris-HCl, $\mathrm{pH} 7.5,2 \mathrm{~h}$ at $\left.37^{\circ} \mathrm{C}\right)$ and pronase $\left(200 \mu \mathrm{g} / \mathrm{mL}, 90 \mathrm{~min}\right.$ at $\left.37^{\circ} \mathrm{C}\right)$, respectively. Samples were mixed with an equal volume ( $8 \mathrm{~mL}$ ) of $8 \%$ SDS and boiled for $15 \mathrm{~min}$ to inactivate the enzymes. Muropeptides were collected by ultracentrifugation and washed 3 times with D.W. to remove SDS from the pellet. The pellet was suspended in $20 \mathrm{mM}$ Tris- $\mathrm{HCl}$ buffer ( $\mathrm{pH} 8.0$ ) and stored at $4{ }^{\circ} \mathrm{C}$ until use. 
Reverse-phase high-performance liquid chromatography (RP-HPLC) analysis was performed as previously described [24] with a few modifications. Total muropeptides were prepared by digesting the purified muropeptides with $20 \mathrm{U}$ mutanolysin (Sigma-Aldrich, St. Louis, MO, USA) at $37^{\circ} \mathrm{C}$ in $20 \mathrm{mM}$ Tris- $\mathrm{HCl}(\mathrm{pH} 8.0)$ for $16 \mathrm{~h}$. The soluble fraction was obtained by centrifugation $(20,000 \times g, 15 \mathrm{~min}$ at 20 ${ }^{\circ} \mathrm{C}$ ) and treated with purified M4Lys $\Delta \mathrm{TMD}$ at $25^{\circ} \mathrm{C}$ overnight. Samples were heat-inactivated for 10 min and reduced with $5 \mathrm{mg}$ sodium borohydride in $500 \mathrm{mM}$ sodium borate buffer (pH 9.0) for $30 \mathrm{~min}$ at room temperature. Borohydride was inactivated by adding $20 \%$ phosphoric acid, the $\mathrm{pH}$ adjusted to 4 , and the sample filtrated using a $0.2 \mu \mathrm{M}$ filter. Prepared samples were loaded onto a Kinetex C18 column $(4.6 \times 250 \mathrm{~mm}, 5 \mathrm{u}$, Phenomenex, Torrance, CA, USA) and a UHPLC C18 column (AJ0-8768, Phenomenex, Torrance, CA, USA) was used as a guard column. RP-HPLC was performed using the Agilent Technologies HPLC 1260 system at the Korea Basic Science Institute (Seoul, South Korea). Samples were injected onto a column preheated to $50^{\circ} \mathrm{C}$ and allowed to bind at a flow rate of $0.5 \mathrm{~mL} / \mathrm{min}$ with a solution of $1 \%$ acetonitrile and $0.1 \%$ trifluoroacetic acid (TFA) for $10 \mathrm{~min}$. A gradient of $1 \%$ to $10 \%$ acetonitrile in $0.1 \%$ TFA solution was used for elution at a flow rate of $0.5 \mathrm{~mL} / \mathrm{min}$ for the next $60 \mathrm{~min}$. Absorbance was detected at $205 \mathrm{~nm}$ and the fractionated muropeptides were identified by matrix-assisted laser desorption/ionization time-of-flight (MALDI-TOF) mass spectrometry.

\subsection{Functional Analysis of the M4Lys TMD}

To understand the role of M4Lys $\triangle T M D$ and the TMD region, each recombinant gene was PCR-amplified, cloned into the pETDuet- 1 dual-expression vector, and expressed with $0.1 \mathrm{mM}$ of IPTG, as described above. The TMD region was truncated from the gene encoding the M4Lys protein with various primers. Primers BSPM4Lys-F-NdeI and BSPM4Lys2-R-XhoI were used to generate the TMD-truncated construct M4Lys $\triangle T M D$. The following three primer sets were used to generate the partially truncated TMD: BSPM4Lys-F-NdeI and BSPM4Lys5_3-R-XhoI for M4Lys 1-224, BSPM4Lys-F-NdeI and BSPM4Lys5_4-R-XhoI for M4Lys ${ }_{1-225}$, and BSPM4Lys-F-NdeI and BSPM4Lys6-R-XhoI for M4Lys ${ }_{1-226}$, respectively. All three PCR-amplified constructs were cloned into the pET28a vector and were transformed into E. coli BL21 (DE3) cells. Then, recombinant plasmid-harboring E. coli cells were used for cell lysis tests with $0.1 \mathrm{mM}$ IPTG induction. Primers used for PCR amplification are listed in Table 2. Experiments for the lysis kinetics were performed in triplicate.

\subsection{Phylogenetic Analysis and Sequence Alignment of M4Lys}

Phylogenetic trees were constructed based on alignment of the M4Lys protein sequence with the amino acid sequences from 37 Gram-negative bacteriophage endolysins available on the NCBI database. Protein sequences were aligned with the Clustal X2 program [25] and the phylogenetic tree was constructed using MEGA7 by the neighbor-joining method with $P$ distance values [26]. Amino acid sequence alignments of endolysins from Chi-like phages were conducted with the Clustal X2 algorithm and edited using the GeneDoc tool [25,27].

\section{Results and Discussion}

\subsection{In Silico Analysis of the BSPM4 Lysis Cassette Showed Two Putative Lysis Genes}

The phage BSPM4 genome sequence includes two putative lysis genes in the predicted lysis cassette, putative lysis protein $\mathrm{B}\left(\mathrm{ORF} \_37\right)$ and putative lysis protein A (ORF_38). A hypothetical gene (ORF_39) was identified downstream of the two putative lysis genes (Figure 1A). Arrangement of the BSPM4 lysis cassette is similar to those of other flagellotropic Salmonella phages such as iEPS5 and FSLSP099 [28,29]. However, the specific function of the putative lysis proteins in host lysis has not yet been characterized. 


\section{A}

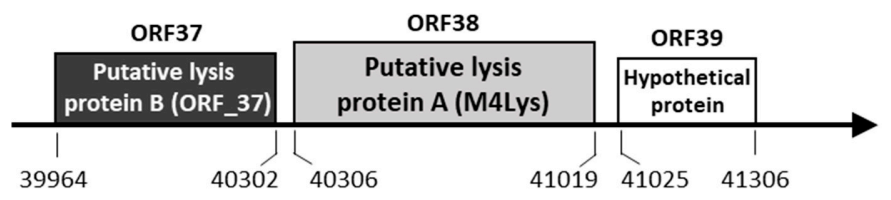

\section{B}

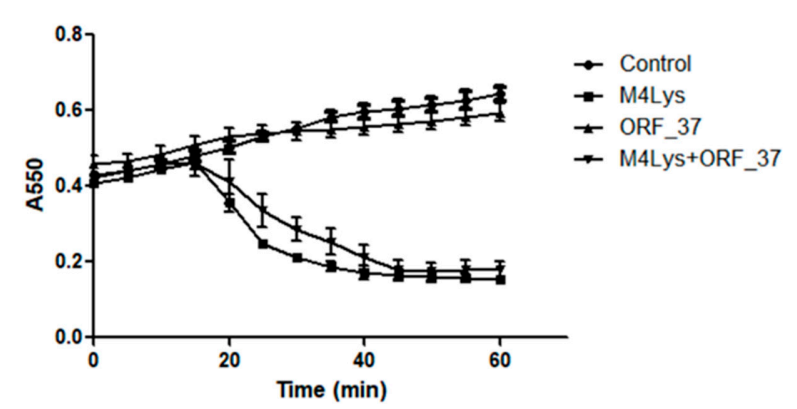

Figure 1. Schematic representation of the phage BSPM4 lysis cassette and analysis of the lysis kinetics by its putative lysis proteins. (A) Schematic representation of the phage BSPM4 lysis cassette; (B) Growth kinetics of the E. coli strains producing the putative lysis protein A (M4Lys) and the putative lysis protein B (ORF_37). Closed circles, empty vector; closed triangles, production of ORF_37; closed squares, production of M4Lys; reverse closed triangles, co-production of ORF_37 and M4Lys. Lysis activity of each gene was investigated by $0.1 \mathrm{mM}$ IPTG induction and the turbidity of the cells was observed. Experiments for the growth kinetics were performed in triplicate. The results of each treatment are represented by the mean \pm standard deviation.

\subsection{Only One Putative Lysis Gene Caused Cell Lysis when Overproduced in E. coli}

In order to understand which gene encodes the BSPM4 phage endolysin, lysis kinetics were examined by overproducing the putative lysis protein B (ORF_37) and the putative lysis protein A (ORF_38) using an E. coli expression system. Endolysin is synthesized and accumulated in the cytosol until the holin creates pores in the inner membrane, thus allowing the endolysin to reach its peptidoglycan substrate target [21,30]. Typically, in vitro production of endolysin alone does not trigger cell lysis [21,31]. However, in our study, E. coli cells were considerably lysed between 20 and $60 \mathrm{~min}$ after putative lysis protein A (M4Lys hereafter) alone was produced by IPTG induction (Figure 1B). Furthermore, M4Lys caused cell lysis more quickly at higher inducer concentrations (data not shown), suggesting that the cell lysis caused by M4Lys is concentration-dependent. Expression of the putative lysis protein B (ORF_37 hereafter) alone showed minimal impact on cell growth compared with the control and did not cause cell lysis (Figure 1B). Coexpression of ORF_37 and M4Lys achieved similar levels of lysis, albeit less rapidly compared with M4Lys expression alone (Figure 1B). Overall, these results suggest that ORF_37 is not essential for host cell lysis in vitro. Slight retardation in lysis rate was observed when the two proteins were produced together compared with M4Lys alone (Figure 1B). We speculate that this could be simply due to the difference in the cellular concentration of M4Lys between the cells producing both M4Lys and ORF_37 proteins simultaneously and those producing M4Lys alone (Figure 1B). However, we cannot exclude the possibility that ORF_37 could control the lysis activity of M4Lys, thus preventing premature lysis.

\subsection{Sec Machinery is Not Involved in M4Lys-Mediated Lysis In Vitro}

Most holin-independent endolysins contain N-terminal signal peptide sequences or SAR sequences essential for their secretion to the periplasm. These sequences often function as secretion sequences for Sec translocase $[8,10,11]$. However, analysis of the M4Lys protein with various bioinformatics tools 
(TargetP V1.0, Psort, DGPI, and SignalP 4.1 [32]) did not reveal any distinct N-terminal signal sequence or SAR sequence in the M4Lys protein sequence. A putative signal peptide cleavage site was detected by SignalP 4.1 only when the settings were optimized to increase sensitivity with a cutoff value of 0.140 (data not shown).

To examine whether Sec machinery is responsible for M4Lys secretion, M4Lys was produced in the presence of $\mathrm{NaN}_{3}$, a well-known inhibitor of E. coli Sec translocase [10,33]. In this experiment, the host cell lysis kinetics of M4Lys was determined by adding various concentrations of $\mathrm{NaN}_{3}$. Increasing concentrations of $\mathrm{NaN}_{3}$ delayed cell lysis by M4LysA but did not inhibit cell lysis (Figure 2). The retardation in cell lysis rate could be due to growth inhibition by high concentrations of $\mathrm{NaN}_{3}$ (Figure 2). This line of evidence implies that the Sec system might not be involved in M4Lys-mediated cell lysis in vitro.

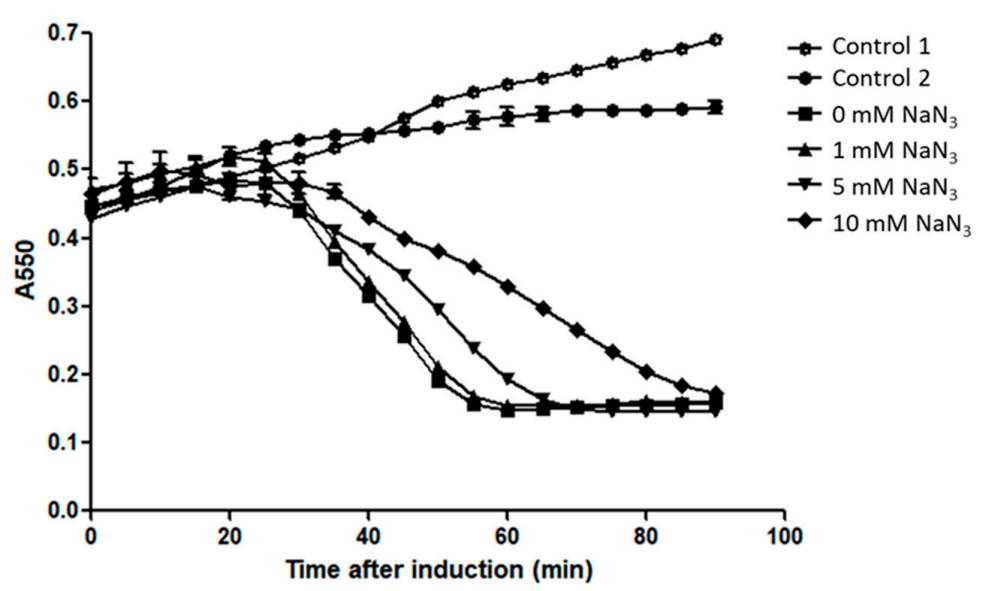

Figure 2. Sec machinery is not involved in M4Lys-mediated cell lysis in vitro. Cell lysis kinetics of M4Lys in the presence of the Sec translocase inhibitor, $\mathrm{NaN}_{3}$. In all experiments, expression of M4Lys from pETDuet-1 plasmid in E. coli cells was induced by adding $0.1 \mathrm{mM}$ of IPTG, except for the controls. Open circles, no $\mathrm{NaN}_{3}$ without IPTG (control 1); closed circles, $10 \mathrm{mM} \mathrm{NaN}_{3}$ without IPTG (control 2); closed squares, no $\mathrm{NaN}_{3}$; closed triangles, $1 \mathrm{mM} \mathrm{NaN}_{3}$; reverse triangles, $5 \mathrm{mM} \mathrm{NaN}_{3}$; and diamonds, $10 \mathrm{mM} \mathrm{NaN}_{3}$. All experiments for the cell lysis kinetics were performed in triplicate and the results of each treatment are represented by the mean \pm standard deviation.

\subsection{Domain Analysis of M4Lys}

Among the 278 endolysins identified in Gram-negative bacteria phages, the majority of endolysins (93.9\%) have a globular structure with a single catalytic domain [34]. The NCBI CDD and sequence search with InterProScan revealed that M4Lys contains a provisional virion protein domain (V superfamily, PHA02564) at the N-terminus (Figure 3A). In addition, Phyre2 analysis revealed that the N-terminus of M4Lys has structural homology (22\% of coverage with $82.1 \%$ confidence) with the peptidoglycan hydrolase (glycosidase) domain from $S$. Typhimurium flagella protein (FlgJ) (data not shown). Since production of the full-length M4Lys protein caused rapid cell lysis (Figure 1B), we speculated that one of these domains, in particular the virion protein domain, could function as a catalytic domain.

A putative TMD was predicted in the C-terminus of M4Lys using Phobius, Phyre2, and TMHMM algorithms [17,35,36] (Figure 3A). Another TMD was detected with only one bioinformatics program (TMHMM) but not with other programs; hence, this second TMD was not included in our study. It is noteworthy that the TMD structure of M4Lys was similar to membrane-spanning transmembrane helices and it contained the highly charged hydrophilic C-terminus found in holins (Figure 3B) [37]. Given our observation that the expression of M4Lys alone caused rapid cell lysis (Figure 1B) in spite of the absence of an obvious signal sequence, we speculated that the TMD could play an important role in M4Lys-mediated cell lysis in vitro. 
A

\begin{tabular}{||l|l|l|l|}
\hline 16 & 157 & 207 & 228237 \\
\hline
\end{tabular}

B

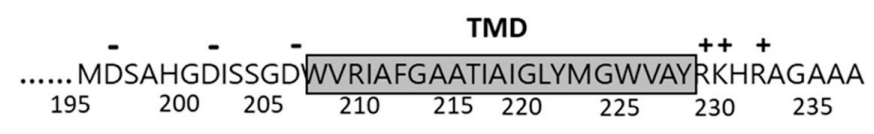

C

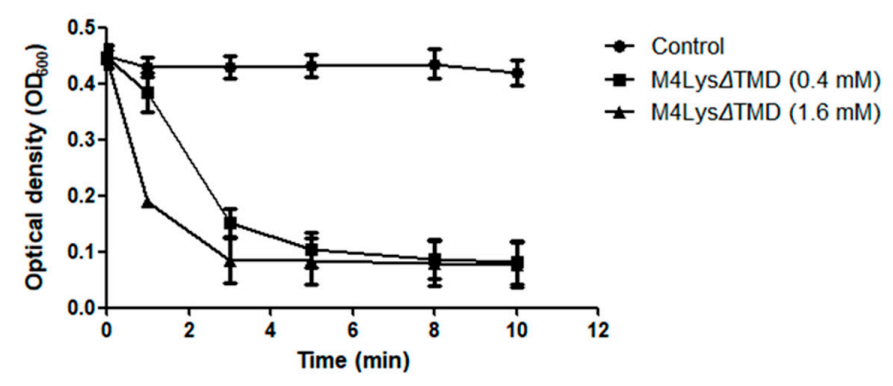

Figure 3. Domain analysis of M4Lys and its peptidoglycan degradation activity. (A) Domain analysis of M4Lys. The numbers represent amino acid positions; (B) Charge distribution of the TMD region of M4Lys. Positive and negative charges are represented as + and -, respectively, above the amino acid sequences. Numbers under the amino acid sequences indicate amino acid position; (C) Turbidity reduction assay of purified M4Lys $\triangle T$ TMD using E. coli peptidoglycan. Closed circles, buffer treatment (control); closed squares, 0.4 mM M4Lys $\Delta$ TMD treatment; and closed triangles, 1.6 mM M4Lys $\Delta$ TMD treatment. Turbidity reduction assays were performed in triplicate and the results of each treatment are represented by the mean \pm standard deviation.

\subsection{Catalytic Activity of M4Lys and Its Target Site Identification}

Peptidoglycan lytic activity of the virion protein domain was assessed by deleting the C-terminal transmembrane domain of M4Lys (M4Lys $\triangle T M D)$. The lytic activity of the full-length M4Lys could not be measured since we failed to purify the protein in spite of repeated attempts, presumably due to the rapid cell lysis that occurred upon its induction. M4Lys $\triangle T M D$ of the predicted size $(24.5 \mathrm{kDa})$ was purified (Figure S1) and incubated with the purified peptidoglycan. Optical density rapidly decreased within $4 \mathrm{~min}$, suggesting that the virion protein domain has a peptidoglycan degradation activity (Figure 3C).

To date, at least five different groups of peptidoglycan catalytic enzymes have been reported, i.e., $N$-acetylmuramidases (lysozymes), endo- $\beta$ - $N$-acetylglucosaminidases (glycosidases), lytic transglycosylases, endopeptidases, and $\mathrm{N}$-acetylmuramoyl L-alanine amidases [38]. To examine the target specificity of the virion protein domain of M4Lys, E. coli peptidoglycan (muropeptides) was predigested with a muramidase (mutanolysin) and incubated with purified M4Lys $\Delta$ TMD. Then, the muropeptides were fractionated by RP-HPLC and analyzed by MS (Figure 4). This analysis revealed that a GlcNAc residue (peaks 4 and 5) was absent both from a monomeric disaccharide-tetrapeptide (Tetra; M4, peak 2) and from a dimer of disaccharide-tetrapeptide (Tetra-Tetra; D44, peak 3) (Figure 4), suggesting $N$-acetylglucosaminidase (glycosidase) activity of M4Lys $\Delta$ TMD. This result again supports the Phyre2 prediction that displayed structural homology between the $\mathrm{N}$-terminus of M4Lys and the glycoside hydrolase domain of the flagella protein FlgJ from Salmonella. Interestingly, endopeptidase activity of M4Lys could also be inferred from the observation that a crosslinked dimer 
of disaccharide-tetrapeptide (peak 3) was reduced and a D-Glu-mDAP crosslink cleaved disaccharide $\left(\mathrm{GM}^{\mathrm{R}}\right.$-Tetra-Di, peak 6) was generated (Figure 4).
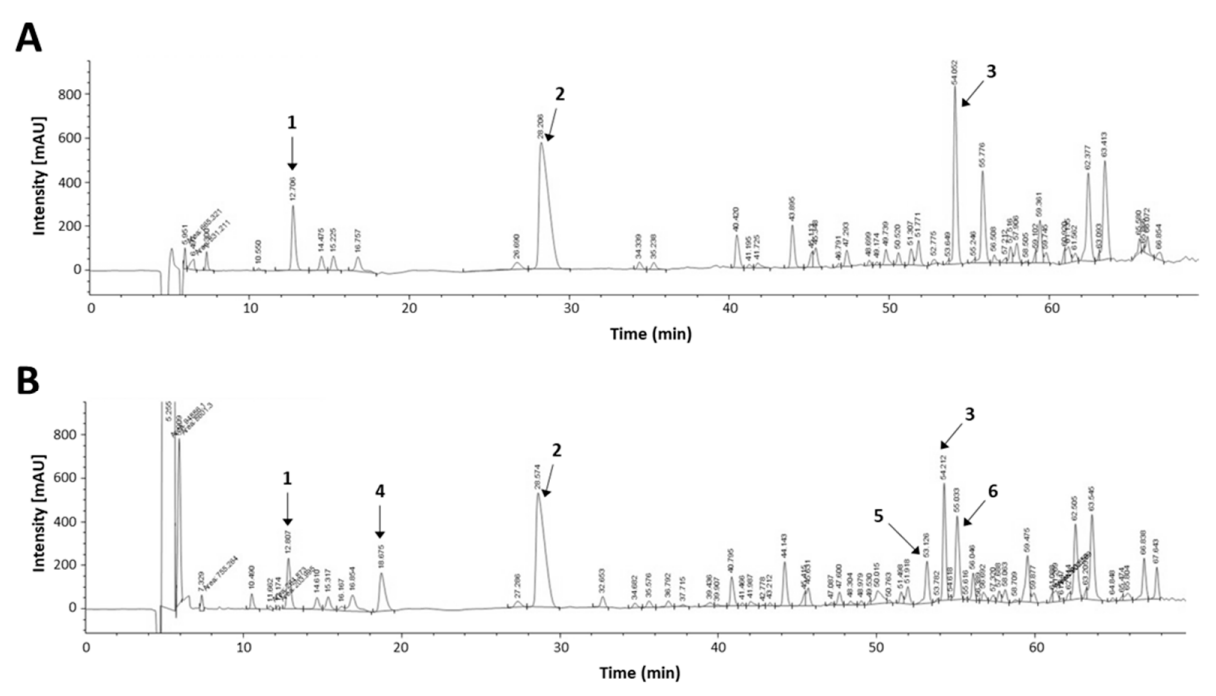

C

\begin{tabular}{|c|c|c|c|c|c|}
\hline \multirow[b]{2}{*}{ Peak } & \multirow[b]{2}{*}{ Inferred structure } & \multirow[b]{2}{*}{$\begin{array}{l}\text { Monoisotopic } \\
\text { Mass (Da) }\end{array}$} & \multicolumn{3}{|c|}{$m / z$} \\
\hline & & & Calculated & $\begin{array}{l}\text { Observed for } \\
\text { mutanolysin }\end{array}$ & $\begin{array}{l}\text { Observed for } \\
\text { M4Lys }\end{array}$ \\
\hline 1 & $\mathrm{GM}^{\mathrm{R}}$-Tri & 870.371 & 871.378 & $893.362^{a}$ & $893.362^{\mathrm{a}}$ \\
\hline 2 & GMR-Tetra & 941.408 & 942.415 & $964.378^{a}$ & $964.368^{\mathrm{a}}$ \\
\hline 3 & $\mathrm{GM}^{\mathrm{R}}$-Tetra-GM $\mathrm{M}^{\mathrm{R}}$-Tetra & 1864.805 & 1865.813 & $1887.765^{a}$ & $1887.748^{a}$ \\
\hline 4 & $M^{R}$-Tetra & 738.330 & 739.335 & ND & $761.350^{\mathrm{a}}$ \\
\hline 5 & $M^{R}$-Tetra-MR-Tetra & 1458.649 & 1459.657 & ND & $1481.582^{a}$ \\
\hline 6 & GMR-Tetra-di & 1185.539 & 1186.546 & ND & 1185.356 \\
\hline
\end{tabular}

Figure 4. Determination of M4Lys cleavage sites. Analysis of E. coli MG1655 peptidoglycan digested by (A) mutanolysin and (B) mutanolysin with purified M4Lys $\triangle T M D$. Soluble muropeptides were reduced and analyzed by RP-HPLC coupled to MS. Peaks corresponding to $\mathrm{m} / \mathrm{z}$ values matching previously identified muropeptides are numbered. The fragmentation pattern (peaks 1,2 , and 3 ) is typical of Tri (L-Ala-D-Glu-m-DAP), Tetra (L-Ala-D-Glu-m-DAP-D-Ala), and Tetra-Tetra muropeptides, respectively. The fragmentation event leading to the loss of a nonreduced GlcNAc residue (203.078, theoretical mass; peaks 4 and 5) indicates the N-acetylmuramidase activity of M4Lys $\Delta$ TMD. Cleavage of D-Glu-mDAP crosslink (peak 6) shows the endopeptidase activity of M4Lys $\triangle T M D$; (C) Inferred structures, theoretical monoisotopic masses, and theoretical and observed $\mathrm{m} / \mathrm{z}$ values of individual peaks are tabulated. $\mathrm{M}^{\mathrm{R}}$, reduced MurNAc; G, GlcNAc; Di, m-DAP (meso-diaminopimelic acid)-D-Ala; Tri, L-Ala-D-Glu-m-DAP; and Tetra, L-Ala-D-Glu-m-DAP-D-Ala.

Endolysins often exhibit a broader antimicrobial spectrum than that of the parental phages [38-41]. Purified M4Lys $\triangle T$ TMD showed a lytic spectrum against various Gram-negative bacteria whereas the parental phage BSPM4 could only infect $S$. Typhimurium strains (Table 3 ).

\subsection{The M4Lys TMD is Important for Cell Lysis by M4Lys In Vitro}

Several lines of evidence previously mentioned suggest that TMD is important for M4Lys-mediated cell lysis in vitro. For instance, in vitro overexpression of M4Lys caused rapid cell lysis (Figure 1B). On the other hand, the expression of M4Lys $\triangle T M D$ alone did not result in cell lysis and the sole expression of TMD exhibited bacteriostatic features (Figure 5A). In addition, expression of M4Lys $\triangle T M D$ with ORF_37 did not trigger cell lysis (Figure 5A). In the topology prediction of TMD using Phobius and Phyre2, the N-terminus region of M4LysA was predicted to face the periplasmic region while the 
C-terminus region was predicted to be localized in the cytoplasmic region (data not shown). Charge distribution of the TMD region also supported the topology prediction results as the C-terminal region of TMD contains three positively charged amino acids, which are sufficient for orientation of the $\mathrm{N}$-terminal region to the periplasm [42] (Figure 3B).
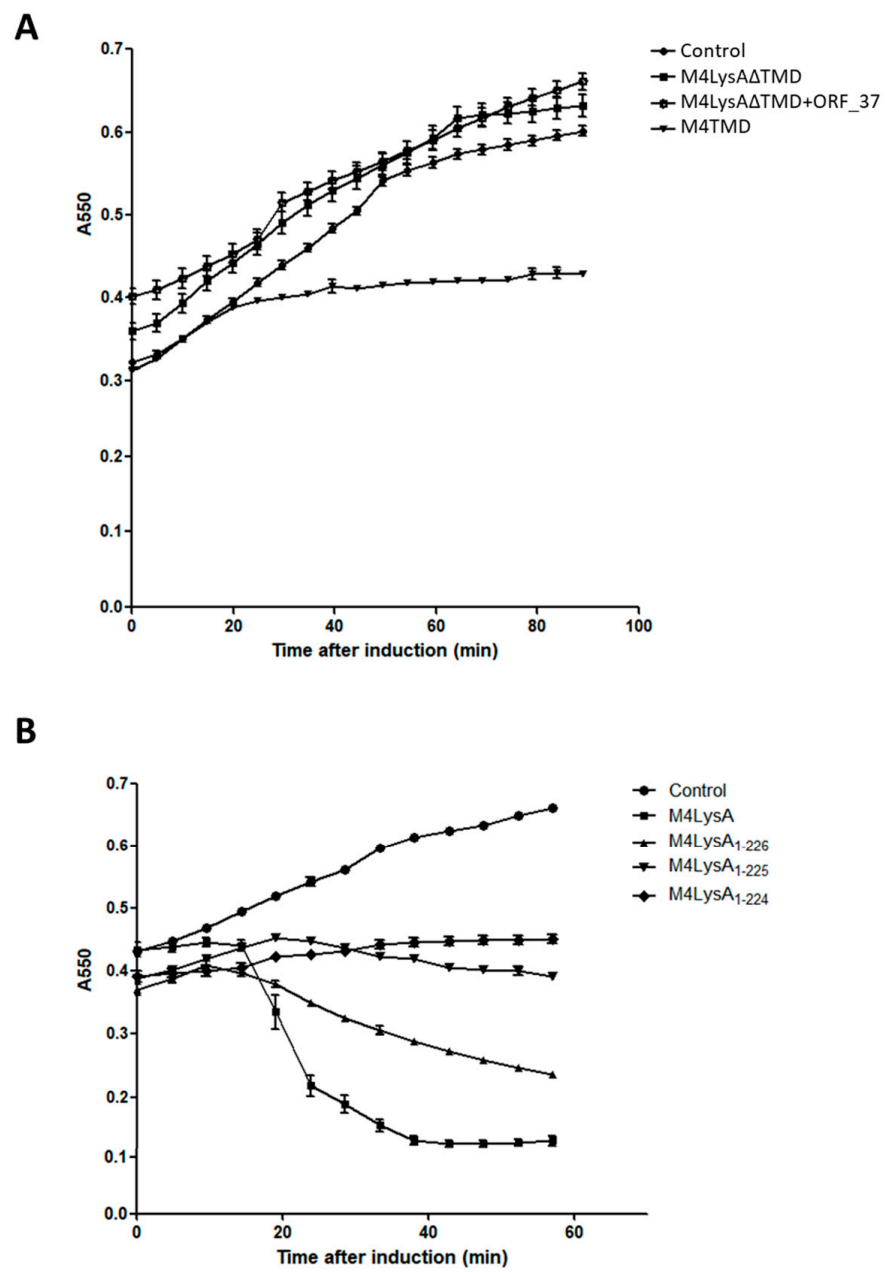

Figure 5. Functional analysis of the M4Lys transmembrane domain. (A) Catalytic domain (M4Lys $\triangle \mathrm{TMD}$ ), C-terminal TMD of M4Lys (M4TMD), and a putative lysis protein B (ORF_37) were produced separately or together in E. coli BL21 (DE3) cells. Closed circles, empty vector; closed square, expression of M4Lys $\triangle T M D$; open square, coexpression of M4Lys $\triangle T M D$ and ORF_37; and reverse closed triangles, expression of TMD; (B) Lysis activity of various deletion mutants compared with the full-length M4Lys. Closed circles, empty vector; closed squares, full-length M4Lys; closed triangles, 11 amino acid deletion in TMD of M4Lys (M4Lys $1-226$ ); reverse closed triangles, 12 amino acid deletion in M4Lys TMD (M4Lys $1-225$ ); and closed diamonds, 13 amino acid deletion of M4Lys TMD (M4Lys $1-224$ ). Truncation of 13 amino acids from the M4Lys sequence eliminated the lytic activity. All protein expression was induced by adding $0.1 \mathrm{mM}$ IPTG. Experiments for cell lysis kinetics were performed in triplicate and the results of each treatment are represented by the mean \pm standard deviation.

The importance of the TMD in cell lysis by M4Lys was reinforced by the change in lytic activity of sequential amino acid truncations of M4Lys C-terminus. Lytic activity further decreased with increasing numbers of amino acid residues eliminated. Eventually, cell lysis activity was virtually

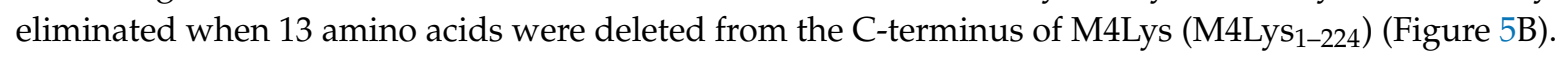

Our current data suggest that the ORF_37 of BSPM4 phage is not required for M4Lys-mediated cell lysis in vitro. However, we cannot currently rule out the possibility that ORF_37 is necessary for optimum cell lysis during BSPM4 phage infection in vivo. 


\subsection{Phylogenetic Analysis of M4Lys}

M4LysA has a unique structure distinct from other canonical endolysins. In order to estimate the evolutionary relationship of M4Lys with other endolysins, phylogenetic analysis was conducted with 37 available endolysin sequences identified from phages infecting diverse Gram-negative bacteria. On the basis of the domain composition, M4Lys was classified as a new group of endolysins (group I) along with those originating from flagella-targeting (Chi-like) phages SPN19, iEPS5, FSLSP088, and Chi (Figure 6A) [28,43-45]. Interestingly, all five phages that belong to group I infect Salmonella. Subsequent BLASTp homology searches and amino acid sequence alignments revealed that the sequence of M4Lys is highly conserved (97\% to 99\% identity) among Chi-like phages (Figure 6B). These results suggest that flagellotropic Chi-like Salmonella phages can share a similar host lysis cassette and that M4Lys is phylogenetically distinct from other endolysins identified from the phages infecting Gram-negative bacteria (Figure 6A).

A

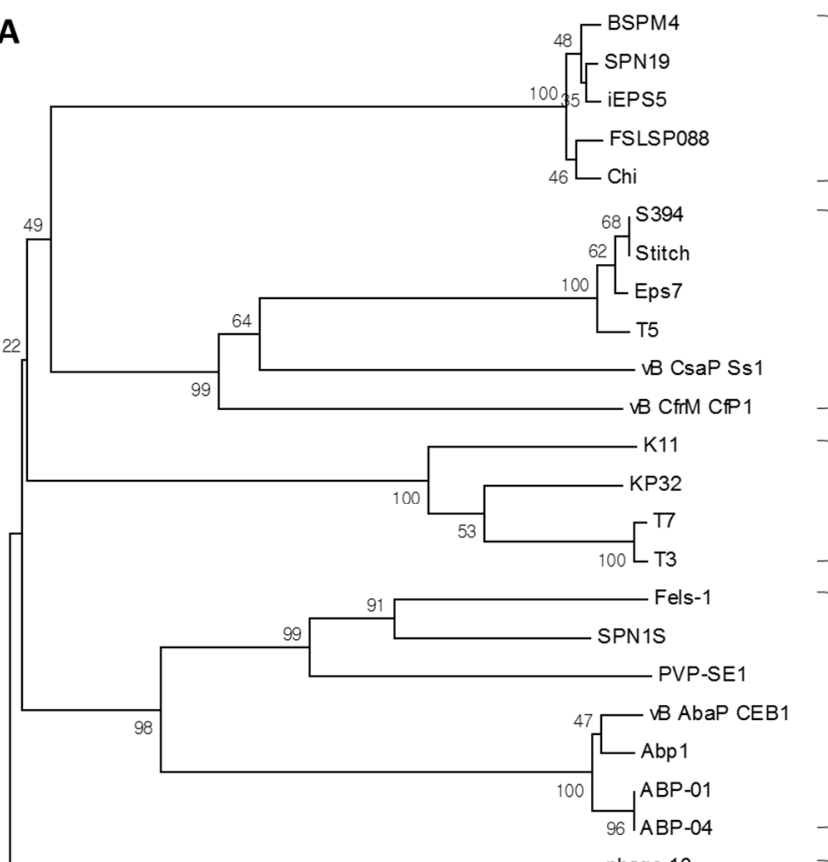

Group I

Group II

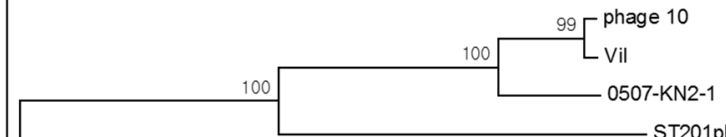

T201phi2-1

40

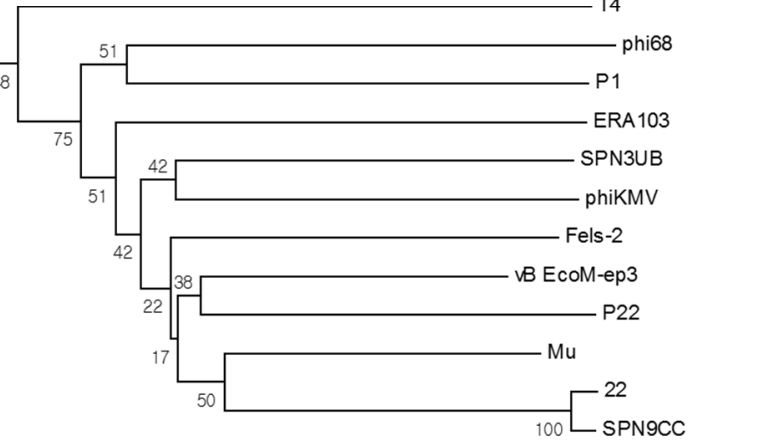

Group III

(1)

Group V

Group VI

Figure 6. Cont. 
B

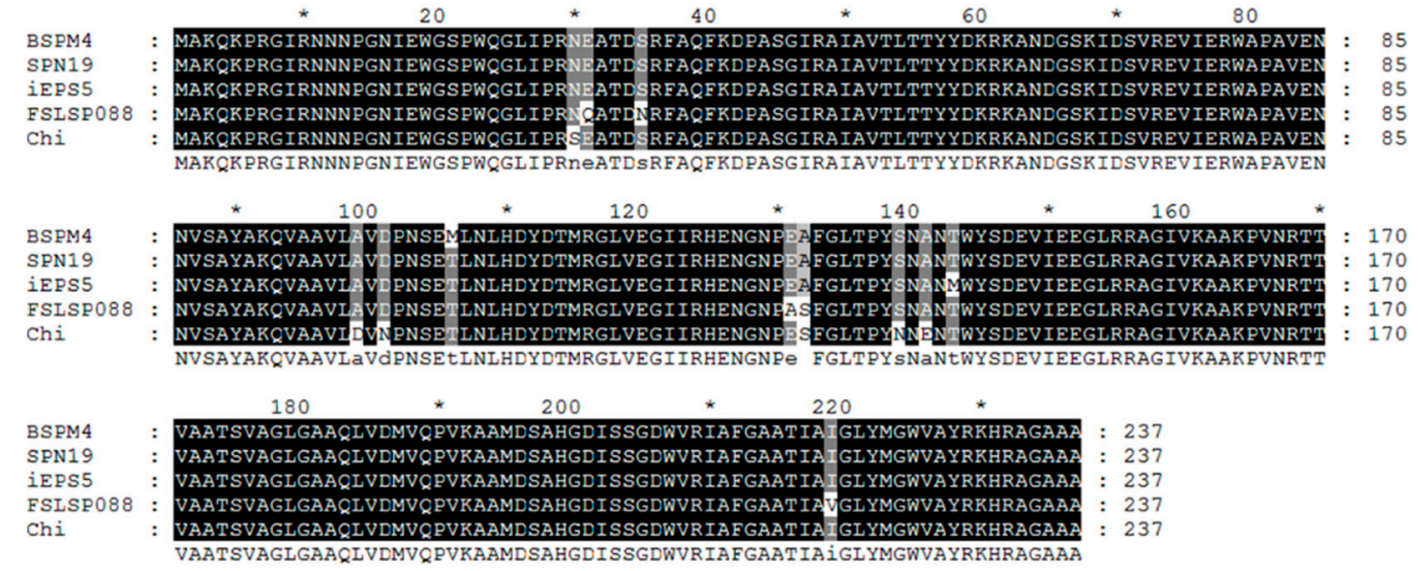

Figure 6. Amino acid sequence alignments of M4Lys with other endolysins. (A) Phylogenetic analysis of endolysins from Gram-negative bacteria phages. Each group corresponds to a different functional domain: group I, V superfamily; group II, D-alanyl-D-alanine-carboxypeptidase; group III, $N$-acetyl muramoyl L-alanine amidase; group IV, glycoside hydrolase family 19; group V, $\mathrm{N}$-acetylmuramidase; and group VI, glycoside hydrolase family 24, respectively; (B) Amino acid sequence alignments of the M4Lys protein of phage BSPM4 with other putative lysis proteins of relevant phages including SPN19, iEPS5, FSLSP088, and Chi. Conserved and identical residues are shaded in gray and black, respectively.

\section{Conclusions}

In this study, we identified and characterized the cell lysis characteristics of M4Lys, a novel endolysin that causes cell lysis when overproduced in vitro. Of particular note is the unusual structure of M4Lys that contains a putative virion protein domain and a TMD. A series of cell lysis kinetic analyses suggested that M4Lys has peptidoglycan degradation activity and that TMD is indispensable for host cell lysis by M4Lys when M4Lys is overexpressed in vitro. This finding enhances our understanding of the host cell lysis mechanism, especially by the flagella-targeting Salmonella phages. However, since this study provided indirect, circumstantial evidence on the potential role of TMD in M4Lys function, further studies are warranted to investigate its specific role in cells. Additionally, we hypothesize that the unique structure of M4Lys could be due to the recombination of two domains and further studies are being conducted in our laboratory to examine the interactions of the two M4Lys domains in an attempt to investigate the mechanism behind the formation of the observed mosaic domain.

Supplementary Materials: The following are available online at http://www.mdpi.com/2076-2607/8/3/447/s1, Figure S1: Expression and purification of M4Lys $\triangle$ TMD.

Author Contributions: J.B. and S.R. conceived and designed the experiments; J.B. performed the experiments; J.B., S.L., and S.R. analyzed the data and wrote the paper. All authors have read and agreed to the published version of the manuscript.

Acknowledgments: This work was supported by the Basic Science Research Programs (NRF-2017R1A2A1A17069378) through the National Research Foundation of Korea (NRF) funded by the Ministry of Science, ICT and Future Planning. J.B. was supported by the BK21 Plus Program of Department of Agricultural Biotechnology, Seoul National University, Seoul, Korea.

Conflicts of Interest: The authors have no competing financial interests to declare.

\section{References}

1. Wang, I.N.; Smith, D.L.; Young, R. Holins: The protein clocks of bacteriophage infections. Annu. Rev. Microbiol. 2000, 54, 799-825. [CrossRef] [PubMed]

2. Bernhardt, T.G.; Roof, W.D.; Young, R. Genetic evidence that the bacteriophage phi X174 lysis protein inhibits cell wall synthesis. Proc. Natl. Acad. Sci. USA 2000, 97, 4297-4302. [CrossRef] [PubMed] 
3. Bernhardt, T.G.; Struck, D.K.; Young, R. The lysis protein E of phi X174 is a specific inhibitor of the MraY-catalyzed step in peptidoglycan synthesis. J. Biol. Chem. 2001, 276, 6093-6097. [CrossRef] [PubMed]

4. Young, I.; Wang, I.; Roof, W.D. Phages will out: Strategies of host cell lysis. Trends Microbiol. 2000, 8, 120-128. [CrossRef]

5. Ackermann, H.W. Bacteriophage observations and evolution. Res. Microbiol. 2003, 154, 245-251. [CrossRef]

6. Xu, M.; Arulandu, A.; Struck, D.K.; Swanson, S.; Sacchettini, J.C.; Young, R. Disulfide isomerization after membrane release of its SAR domain activates P1 lysozyme. Science 2005, 307, 113-117. [CrossRef]

7. Young, R. Bacteriophage lysis: Mechanism and regulation. Microbiol. Rev. 1992, 56, 430-481. [CrossRef]

8. Xu, M.; Struck, D.K.; Deaton, J.; Wang, I.N.; Young, R. A signal-arrest-release sequence mediates export and control of the phage P1 endolysin. Proc. Natl. Acad. Sci. USA 2004, 101, 6415-6420. [CrossRef]

9. Sun, Q.; Kuty, G.F.; Arockiasamy, A.; Xu, M.; Young, R.; Sacchettini, J.C. Regulation of a muralytic enzyme by dynamic membrane topology. Nat. Struct. Mol. Biol. 2009, 16, 1192-1194. [CrossRef]

10. Briers, Y.; Peeters, L.M.; Volckaert, G.; Lavigne, R. The lysis cassette of bacteriophage phiKMV encodes a signal-arrest-release endolysin and a pinholin. Bacteriophage 2011, 1, 25-30. [CrossRef]

11. Sao-Jose, C.; Parreira, R.; Vieira, G.; Santos, M.A. The N-terminal region of the Oenococcus oeni bacteriophage fOg44 lysin behaves as a bona fide signal peptide in Escherichia coli and as a cis-inhibitory element, preventing lytic activity on oenococcal cells. J. Bacteriol. 2000, 182, 5823-5831. [CrossRef] [PubMed]

12. Catalao, M.J.; Gil, F.; Moniz-Pereira, J.; Pimentel, M. The mycobacteriophage Ms6 encodes a chaperone-like protein involved in the endolysin delivery to the peptidoglycan. Mol. Microbiol. 2010, 77, 672-686. [CrossRef] [PubMed]

13. Bai, J.; Jeon, B.; Ryu, S. Effective inhibition of Salmonella Typhimurium in fresh produce by a phage cocktail targeting multiple host receptors. Food Microbiol. 2019, 77, 52-60. [CrossRef] [PubMed]

14. Altschul, S.F.; Madden, T.L.; Schaffer, A.A.; Zhang, J.; Zhang, Z.; Miller, W.; Lipman, D.J. Gapped BLAST and PSI-BLAST: A new generation of protein database search programs. Nucleic Acids Res. 1997, 25, 3389-3402. [CrossRef] [PubMed]

15. Quevillon, E.; Silventoinen, V.; Pillai, S.; Harte, N.; Mulder, N.; Apweiler, R.; Lopez, R. InterProScan: Protein domains identifier. Nucleic Acids Res. 2005, 33, W116-W120. [CrossRef] [PubMed]

16. Marchler-Bauer, A.; Anderson, J.B.; Derbyshire, M.K.; DeWeese-Scott, C.; Gonzales, N.R.; Gwadz, M.; Hao, L.; He, S.; Hurwitz, D.I.; Jackson, J.D.; et al. CDD: A conserved domain database for interactive domain family analysis. Nucleic Acids Res. 2007, 35, D237-D240. [CrossRef]

17. Kall, L.; Krogh, A.; Sonnhammer, E.L. Advantages of combined transmembrane topology and signal peptide prediction-the Phobius web server. Nucleic Acids Res. 2007, 35, W429-W432. [CrossRef]

18. Studier, F.W.; Moffatt, B.A. Use of Bacteriophage-T7 Rna-Polymerase to Direct Selective High-Level Expression of Cloned Genes. J. Mol. Biol. 1986, 189, 113-130. [CrossRef]

19. Chang, Y.; Ryu, S. Characterization of a novel cell wall binding domain-containing Staphylococcus aureus endolysin LysSA97. Appl. Microbiol. Biotechnol. 2017, 101, 147-158. [CrossRef]

20. Fein, J.E.; Rogers, H.J. Autolytic enzyme-deficient mutants of Bacillus subtilis 168. J. Bacteriol. 1976, 127, 1427-1442. [CrossRef]

21. Lim, J.A.; Shin, H.; Kang, D.H.; Ryu, S. Characterization of endolysin from a Salmonella Typhimurium-infecting bacteriophage SPN1S. Res. Microbiol. 2012, 163, 233-241. [CrossRef]

22. Lee, J.H.; Bai, J.; Shin, H.; Kim, Y.; Park, B.; Heu, S.; Ryu, S. A Novel Bacteriophage Targeting Cronobacter sakazakii Is a Potential Biocontrol Agent in Foods. Appl. Environ. Microbiol. 2016, 82, 192-201. [CrossRef]

23. Desmarais, S.M.; Cava, F.; de Pedro, M.A.; Huang, K.C. Isolation and preparation of bacterial cell walls for compositional analysis by ultra performance liquid chromatography. J. Vis. Exp. 2014, 83, e51183. [CrossRef]

24. Singh, S.K.; SaiSree, L.; Amrutha, R.N.; Reddy, M. Three redundant murein endopeptidases catalyse an essential cleavage step in peptidoglycan synthesis of E scherichia coli K 12. Mol. Microbiol. 2012, 86, 1036-1051. [CrossRef]

25. Larkin, M.A.; Blackshields, G.; Brown, N.P.; Chenna, R.; McGettigan, P.A.; McWilliam, H.; Valentin, F.; Wallace, I.M.; Wilm, A.; Lopez, R.; et al. Clustal W and Clustal X version 2.0. Bioinformatics 2007, 23, 2947-2948. [CrossRef]

26. Kumar, S.; Stecher, G.; Tamura, K. MEGA7: Molecular Evolutionary Genetics Analysis Version 7.0 for Bigger Datasets. Mol. Biol. Evol. 2016, 33, 1870-1874. [CrossRef] 
27. Nicholas, K.B.; Nicholas, H.J. GeneDoc: A Tool for Editing and Annotating Multiple Sequence Alignments. Available online: http://www.psc.edu/biomed/genedoc (accessed on 17 February 2017).

28. Choi, Y.; Shin, H.; Lee, J.H.; Ryu, S. Identification and characterization of a novel flagellum-dependent Salmonella-infecting bacteriophage, iEPS5. Appl. Environ. Microbiol. 2013, 79, 4829-4837. [CrossRef]

29. Moreno Switt, A.I.; Orsi, R.H.; den Bakker, H.C.; Vongkamjan, K.; Altier, C.; Wiedmann, M. Genomic characterization provides new insight into Salmonella phage diversity. BMC Genom. 2013, 14, 481. [CrossRef]

30. Chang, Y.; Yoon, H.; Kang, D.H.; Chang, P.S.; Ryu, S. Endolysin LysSA97 is synergistic with carvacrol in controlling Staphylococcus aureus in foods. Int. J. Food Microbiol. 2017, 244, 19-26. [CrossRef]

31. Fernandes, S.; Sao-Jose, C. More than a hole: The holin lethal function may be required to fully sensitize bacteria to the lytic action of canonical endolysins. Mol. Microbiol. 2016, 102, 92-106. [CrossRef]

32. Petersen, T.N.; Brunak, S.; von Heijne, G.; Nielsen, H. SignalP 4.0: Discriminating signal peptides from transmembrane regions. Nat. Methods 2011, 8, 785-786. [CrossRef]

33. Valent, Q.A. Signal recognition particle mediated protein targeting in Escherichia coli. Antonie Van Leeuwenhoek 2001, 79, 17-31. [CrossRef]

34. Briers, Y.; Lavigne, R. Breaking barriers: Expansion of the use of endolysins as novel antibacterials against Gram-negative bacteria. Future Microbiol. 2015, 10, 377-390. [CrossRef]

35. Kelley, L.A.; Mezulis, S.; Yates, C.M.; Wass, M.N.; Sternberg, M.J. The Phyre2 web portal for protein modeling, prediction and analysis. Nat. Protoc. 2015, 10, 845-858. [CrossRef]

36. Server, T.V. 2.0. TMHMM. Available online: http://www.cbs.dtu.dk/services (accessed on 31 January 2019).

37. Young, R.; Blasi, U. Holins: Form and function in bacteriophage lysis. FEMS Microbiol. Rev. 1995, 17, 191-205. [CrossRef]

38. Borysowski, J.; Weber-Dabrowska, B.; Gorski, A. Bacteriophage endolysins as a novel class of antibacterial agents. Exp. Biol. Med. 2006, 231, 366-377. [CrossRef]

39. Zhang, W.H.; Mi, Z.Q.; Yin, X.Y.; Fan, H.; An, X.P.; Zhang, Z.Y.; Chen, J.K.; Tong, Y.G. Characterization of Enterococcus faecalis Phage IME-EF1 and Its Endolysin. PLoS ONE 2013, 8, e80435. [CrossRef]

40. Yuan, Y.H.; Peng, Q.; Gao, M.Y. Characteristics of a broad lytic spectrum endolysin from phage BtCS33 of Bacillus thuringiensis. BMC Microbiol. 2012, 12, 297. [CrossRef]

41. Kong, M.; Ryu, S. Bacteriophage PBC1 and Its Endolysin as an Antimicrobial Agent against Bacillus cereus. Appl. Environ. Microbiol. 2015, 81, 2274-2283. [CrossRef]

42. Whitley, P.; Gafvelin, G.; Vonheijne, G. Seca-Independent Translocation of the Periplasmic N-Terminal Tail of an Escherichia-Coli Inner Membrane-Protein-Position-Specific Effects on Translocation of Positively Charged Residues and Construction of a Protein with a C-Terminal Translocation Signal. J. Biol. Chem. 1995, 270, 29831-29835.

43. Lee, J.H.; Shin, H.; Choi, Y.; Ryu, S. Complete genome sequence analysis of bacterial-flagellum-targeting bacteriophage chi. Arch. Virol. 2013, 158, 2179-2183. [CrossRef]

44. Shin, H.; Lee, J.H.; Kim, H.; Choi, Y.; Heu, S.; Ryu, S. Receptor diversity and host interaction of bacteriophages infecting Salmonella enterica serovar Typhimurium. PLoS ONE 2012, 7, e43392. [CrossRef]

45. Phothaworn, P.; Dunne, M.; Supokaivanich, R.; Ong, C.; Lim, J.; Taharnklaew, R.; Vesaratchavest, M.; Khumthong, R.; Pringsulaka, O.; Ajawatanawong, P.; et al. Characterization of Flagellotropic, Chi-Like Salmonella Phages Isolated from Thai Poultry Farms. Viruses 2019, 11, 520. [CrossRef]

(C) 2020 by the authors. Licensee MDPI, Basel, Switzerland. This article is an open access article distributed under the terms and conditions of the Creative Commons Attribution (CC BY) license (http://creativecommons.org/licenses/by/4.0/). 